\title{
A Case of Hereditary Hemorrhagic Telangiectasia
}

\author{
Ha Eun Lee, M.D., Chan Sagong, M.D., Kwang Yeoll Yeo, M.D., \\ Joo Yeon Ko, M.D., Joung Soo Kim, M.D., Hee Joon Yu, M.D. \\ Department of Dermatology, Hanyang University College of Medicine, Seoul, Korea
}

Hereditary hemorrhagic telangiectasia, also known as Osler-Weber-Rendu disease, is an autosomal dominant disorder of the fibrovascular tissue. It is characterized by the classic triad of mucocutaneous telangiectasias, recurrent hemorrhages, and familial occurrence. The cutaneous manifestation appear clinically as punctuate, linear, or splinter-like telangiectasias of the upper body, oral, and nasal mucous membranes, and nail beds. A 73-year-old woman presented with purpuric, punctuate, and tiny macules on the finger tips of both hands and the tongue. The skin lesions were discovered about 50 years previously. She had a family history of cutaneous telangiectasia. Also, she had episodes of recurrent epistaxis, gastrointestinal bleeding, and anemia. The gastroendoscopy revealed gastric angiodysplasia of the fundus and body of the stomach. The histopathologic study showed dilated capillaries lined by flat endothelial cells in the papillary dermis. From these findings, we diagnosed this case as hereditary hemorrhagic telangiectasia, which has rarely been reported in the dermatologic literature. (Ann Dermatol 21(2) 206 208, 2009)

\section{-Keywords-}

Hereditary hemorrhagic telangiectasia, Osler-Weber-Rendu disease

\section{INTRODUCTION}

Hereditary hemorrhagic telangiectasia $(\mathrm{HHT})$, also known as Osler-Weber-Rendu disease, is an autosomal dominant disorder of the fibrovascular tissue ${ }^{1}$. It is characterized by

Received October 21, 2008, Accepted for publication November 28, 2008

Reprint request to: Hee Joon Yu, M.D., Department of Dermatology, Hanyang University Guri Hospital, 249-1, Gyomun-dong, Guri 471-701, Korea. Tel: 82-31-560-2285, Fax: 82-31-557-4872, E-mail: yuhjoon@hanyang.ac.kr the classic triad of mucocutaneous telangiectasias, arteriovenous malformations with recurrent epistaxis and hemorrhages, and inheritance ${ }^{2}$. Clinically it appears as punctuate or splinter-like telangiectasias located on the lips, oral mucosa, upper extremities, nail beds, and trunk ${ }^{3}$. HHT has rarely been reported in the dermatologic literature. We report a typical case of HHT in a 73-year-old female who had recurrent epistaxis and several skin and visceral manifestations.

\section{CASE REPORT}

A 73-year-old woman was referred for evaluation of purpuric, punctuate, and tiny macules on the finger tips of both hands and the tongue which had been present for 50 years (Fig. 1). She had suffered numerous episodes of mild-to-severe nasal bleeding of unknown cause. She had been treated for epistaxis with electrocauterization therapy several times. She had a history of admissions to the Department of Gastroenterology due to exacerbations of anemia and melena 2 years before. During her cutaneous examination, we noticed pallor and discoloration with telangiectasias in the oral mucosa and tongue. The family history was significant for recurrent epistaxis and telangiectatic lesions in her mother and two sisters. No abnormalities were detected on chest $\mathrm{x}$-ray. The laboratory work-up at admission revealed the following: WBC, $2,500 / \mathrm{mm}^{3}$; platelet count, $207,000 / \mathrm{mm}^{3}$; hemoglobin, $6.5 \mathrm{~g} / \mathrm{dl}$; ferritin, $3.72(13 \sim 150 \mathrm{ng} / \mathrm{ml})$; and serum iron 34 (50 $150 \mathrm{ug} / \mathrm{dl})$. Other laboratory work-up was normal, including bleeding time, coagulation time, prothrombin time (PT), activated partial thromboplastin time (aPTT), and stool occult blood. Endoscopy of the upper digestive tract was performed. The results of the endoscopy indicated multiple gastric angiodysplasias of the fundus and body of the stomach (Fig. 2). To prevent continuous bleeding and correction of chronic anemia, hemoclipping of multiple vascular lesions of the GI tract was performed as 

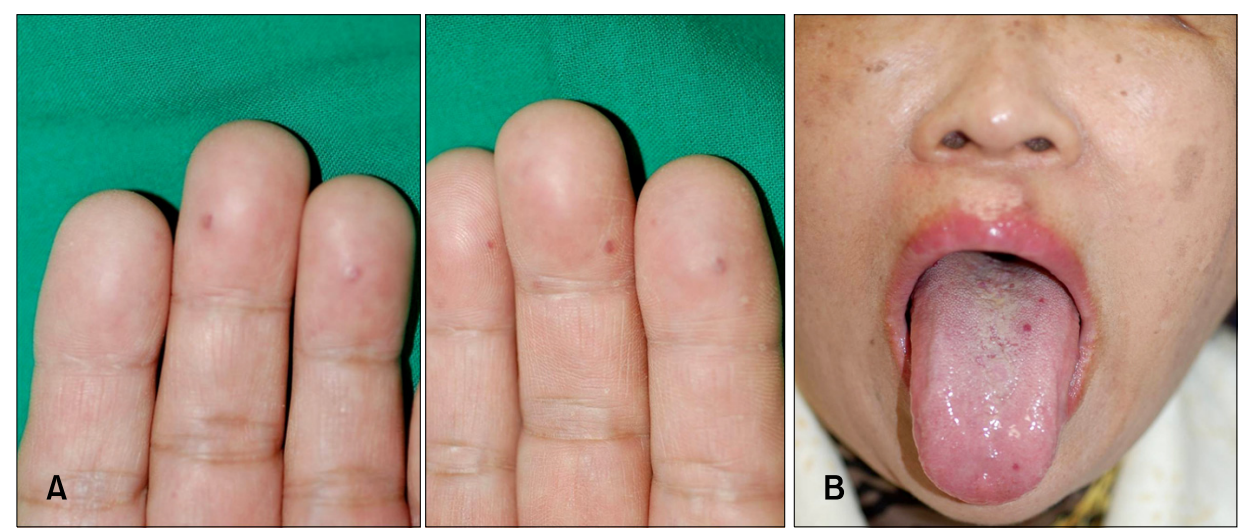

Fig. 1. Typical punctuate or splinterlike telangiectasias on the (A) fingertips (B) and tongue.

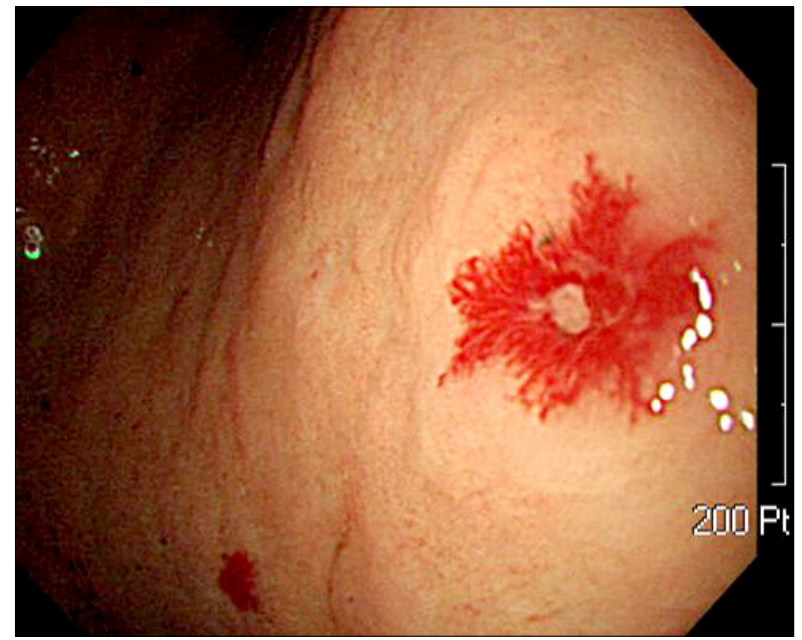

Fig. 2. HHT manifestation in the Gl tract with angiodysplasia in the fundus of the stomach.

palliative treatment. We performed a punch biopsy from one of the macules on her left finger tip. The histopathologic study showed dilated capillaries lined by flat endothelial cells in the papillary dermis (Fig. 3). From these findings, the diagnosis of hereditary hemorrhagic telangiectasia was made.

\section{DISCUSSION}

HHT is a hereditary disorder with autosomal dominant transmission, despite the fact that about $20 \%$ of the cases do not have a family history. The reported incidence of HHT is approximately 1 per 5,000 10,000 population per year ${ }^{1}$. It is thought that the abnormal vessels in HHT develop because of aberrant TGF signaling at some stage during vascular development and homeostasis due to mutations of HHT-associated genes. There are two major types of HHT (HHT1 and HHT2). It has been proposed that in the case of $\mathrm{HHT}$, disease severity is more pro-

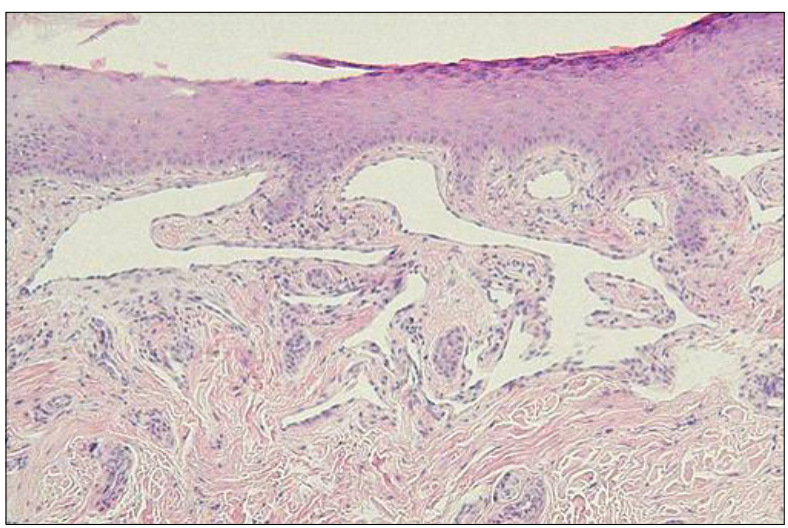

Fig. 3. The histopathologic findings from the papules on the fingertip show dilated capillaries lined by flat endothelial cells in the papillary dermis $(H \& E, \times 100)$.

nounced in $\mathrm{HHT} 1$ compared to $\mathrm{HHT} 2$, with an earlier age of onset for epistaxis, the appearance of telangiectasias, and a higher incidence of pulmonary $\mathrm{AVMs}^{4}$. HHT1 can be induced by mutations in the gene, ENG (endoglin), encoding endoglin on chromosome $9 q^{33,34}$. HHT2 can be induced by mutations in the gene, ALK-1 (activin receptor-like kinase 1), encoding activin receptor-like kinase 1 on chromosome $12 q 13^{5}$. These events cause alteration in the elastic and muscle layers of vessel walls, making them more vulnerable to spontaneous rupture and injuries ${ }^{6}$.

The diagnosis of HHT is established when three of the following features are present: (1) epistaxis (spontaneous, recurrent nose bleeds); (2) multiple telangiectasias at characteristic sites (lips, oral cavity, fingers, and nose); (3) visceral lesions, such as gastrointestinal telangiectasia (with or without bleeding), pulmonary arteriovascular malformation (AVM), hepatic AVM, cerebral AVM, spinal AVM; and (4) family history (a first-degree relative with $\mathrm{HHT}^{7}$. Our case met four criteria for HHT: recurrent epistaxis, telangiectasias of the fingertips and tongue, GI bleeding combined with anemia, and first-degree familial tendency. 
The clinical manifestations of HHT are known to be variable and age-dependent ${ }^{1}$. Epistaxis is the first manifestation and the most common symptom, but patients may have a variety of serious complications due to vascular involvement of internal organs, such as the gastrointestinal tract, the lungs, and the central nervous system. In the follow-up of affected persons, the lung and brain are of particular concern, because each may contain clinically silent lesions that can result in sudden morbidity or death. Pulmonary AVMs, such as right-to-left shunts can result in hypoxemia. Furthermore, the absence of a filtering capillary bed allows emboli to reach the systemic circulation, which may cause cerebral abscesses and stroke. Cerebral AVMs can lead to headaches, migraines, brain abcesses, seizures, paraparesis, ischemia, strokes, transient ischemic attacks, and both intracerebral and subarachnoid hemorrhage. Gastrointestinal bleeding can result in iron deficiency anemia or acute gastrointestinal hemorrhage ${ }^{1}$. Vascular lesions may be present as telangiectasias, arteriovenous malformations (AVM), or aneurysms.

Treatment options for HHT should be considered individually for each patient, owing to the diverse clinical manifestations of this disease ${ }^{1}$. Therapy for the bleeding is primarily supportive and palliative. Telangiectasias of the skin and the mucosa are not merely a cosmetic problem, but may cause hemorrhages in $>27 \%$ of patients, mostly involving hemorrhage from the tongue, the fingers, and the skin of the supraclavicular fossa, which worsen with age and alter the quality of life. Treatment should be offered to these patients. However, the awareness of the therapeutic necessities and specific methods of treatment were not adequate until now. Management options for cutaneous lesions include electrocauterization with diathermy, sclerotherapy, or laser therapy. Many different types of laser therapy have been used to minimize and/or eliminate these telangiectasias, such as Nd:YAG, IPL, argon, and the tunable dye lasers, all of which have been reported to be effective ${ }^{8}$.

Presymptomatic intervention in HHT may substantially affect the outcome. An early diagnosis is essential in high-risk individuals in order to alter their clinical course and prognosis. Patients with a family history of HHT with pulmonary disease are at high risk of having pulmonary AVMs. Chest CT-scan must be used and pulmonary angiography should be used for radiologic or surgical treatment planning. MRI is useful for assessing CNS involve- ment $^{7}$.

Skin manifestations could be regarded as a minor condition of $\mathrm{HHT}$, but the capacity for stigmatization must not be underestimated. It is important to recognize the skin manifestation of HHT for the early diagnosis of HHT to prevent complications. Moreover dermatologists should consider clinically similar telangiectactic diseases, including CREST syndrome (calcinosis cutis, Raynaud's phenomenon, esophageal dysfunction, sclerodactyly, and telangi-

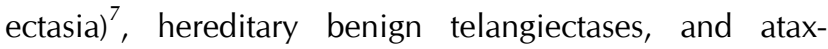
ia-telangiectasia as the differential diagnosis for HHT. Greater clinical awareness for dermatologists may be the first step to identify patients with HHT, and as such, may play a key role in the timely referral of these patients and their family members.

\section{REFERENCES}

1. te Veldhuis EC, te Veldhuis $A H$, van Dijk FS, Kwee ML, van Hagen JM, Baart JA, et al. Rendu-Osler-Weber disease: update of medical and dental considerations. Oral Surg Oral Med Oral Pathol Oral Radiol Endod 2008;105:e38-e41.

2. Sadick H, Sadick M, Gotte K, Naim R, Riedel F, Bran G, et al. Hereditary hemorrhagic telangiectasia: an update on clinical manifestations and diagnostic measures. Wien Klin Wochenschr 2006;118:72-80.

3. Chang DS, Kim MN, Hong CK, Ro BI. A case of hereditary hemorrhagic telangiectasia. Korean J Dermatol 2002;40: 1148-1150.

4. Bossler AD, Richards J, George C, Godmilow L, Ganguly A. Novel mutations in ENG and ACVRL1 identified in a series of 200 individuals undergoing clinical genetic testing for hereditary hemorrhagic telangiectasia (HHT): correlation of genotype with phenotype. Hum Mutat 2006;27:667-675.

5. Abdalla SA, Letarte M. Hereditary haemorrhagic telangiectasia: current views on genetics and mechanisms of disease. J Med Genet 2006;43:97-110.

6. Juares AJ, Dell'Aringa AR, Nardi JC, Kobari K, Gradim Moron Rodrigues VL, Perches Filho RM. Rendu-Osler-Weber Syndrome: case report and literature review. Braz J Otorhinolaryngol 2008;74:452-457.

7. Garzon MC, Huang JT, Enjolras O, Frieden IJ. Vascular malformations. Part II: associated syndromes. J Am Acad Dermatol 2007;56:541-564.

8. Fernandez-Jorge B, Del Pozo Losada J, Paradela S, MartinezGonzalez C. Treatment of cutaneous and mucosal telangiectases in hereditary hemorrhagic telangiectasia: report of three cases. J Cosmet Laser Ther 2007;9:29-33. 